\title{
Publisher Correction: Exosomes maintain cellular homeostasis by excreting harmful DNA from cells
}

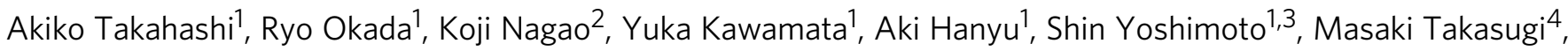 \\ Sugiko Watanabe ${ }^{4}$, Masato T. Kanemaki ${ }^{5,6}$, Chikashi Obuse ${ }^{2}$ \& Eiji Hara ${ }^{1,4,7}$
}

Correction to: Nature Communications; https://doi.org/10.1038/ncomms15287; published online 16 May 2017.

This Article contains errors in Fig. 4. In panel d, the lanes of the western blot should have been labeled '1.05', '1.06, '1.09', '1.11' ' 1.13 ', '1.16', '1.19', '1.22', '1.24', '1.25'. The correct version of Fig. 4 appears below.

Published online: 08 October 2018

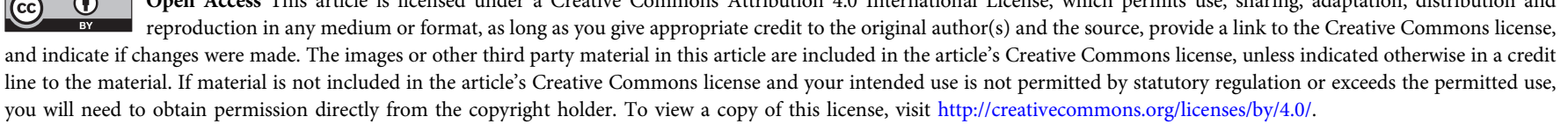

(C) The Author(s) 2018

\footnotetext{
${ }^{1}$ The Cancer Institute, Japanese Foundation for Cancer Research (JFCR), Koto-ku, Tokyo 135-8550, Japan. ${ }^{2}$ Graduate School of Life Science, Hokkaido University, Sapporo, Hokkaido 001-0021, Japan. ${ }^{3}$ LSI Medience Corporation, Chiyoda-ku, Tokyo 101-8517, Japan. ${ }^{4}$ Department of Molecular Microbiology, Research Institute for Microbial Diseases (RIMD), Osaka University, Suita, Osaka 565-0871, Japan. ${ }^{5}$ Division of Molecular Cell Engineering, Department of Genetics, National Institute of Genetics, ROIS, SOKENDAI, Mishima, Shizuoka 411-8540, Japan. 6 PRESTO, Japan Science and Technology Agency (JST), Kawaguchi, Saitama 332-0012, Japan. ${ }^{7}$ CREST, Japan Agency for Medical Research and Development (AMED), Chiyoda-ku, Tokyo 100-0004, Japan. Correspondence and requests for materials should be addressed to A.T. (email: akiko.takahashi@jfcr.or.jp) or to E.H. (email: ehara@biken.osaka-u.ac.jp)
} 
a

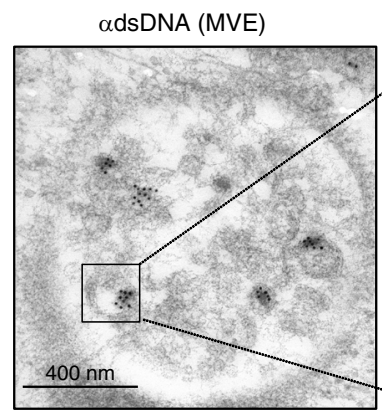

$\alpha$ dsDNA (Exosome)

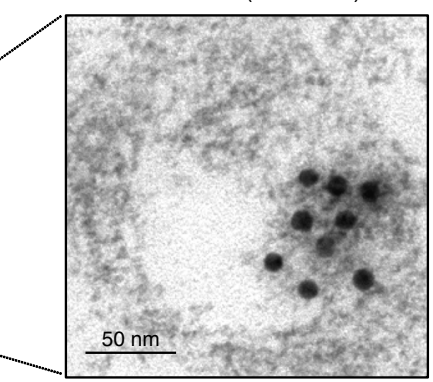

b

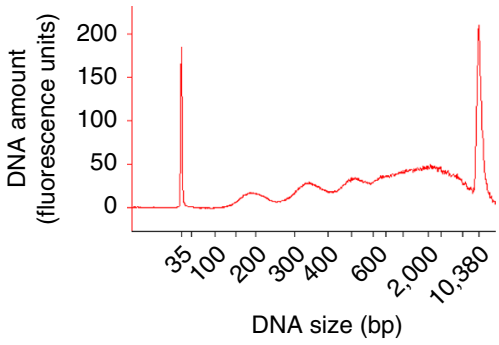

C

Genomic DNA

(RPKM)

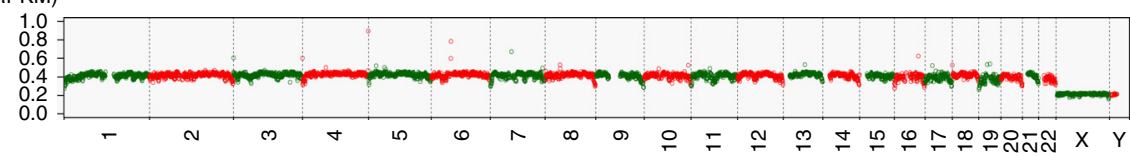

(RPKM)

Exosomal DNA

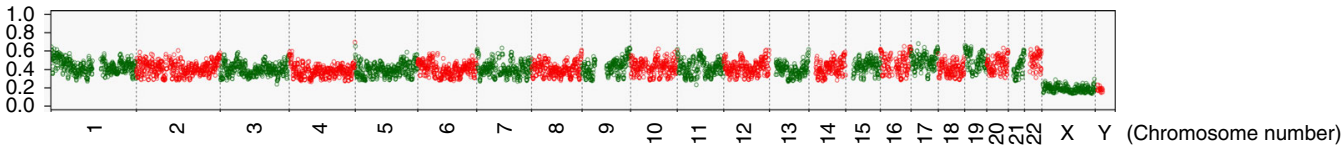

d

Density
$\left(\mathrm{g} \mathrm{ml}^{-1}\right)$
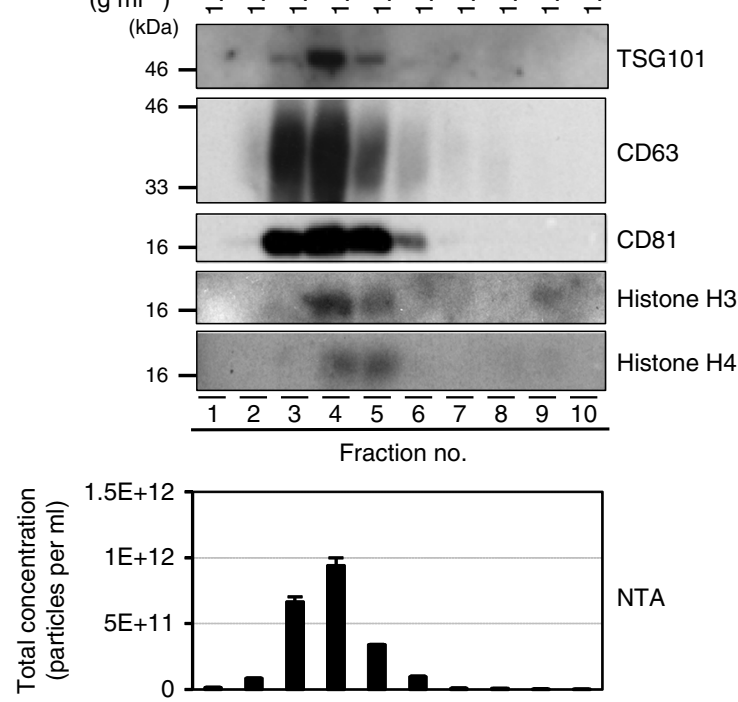

NTA

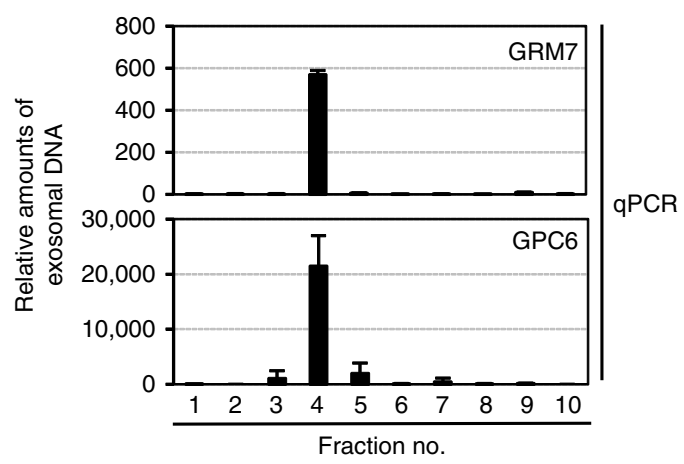

Fig. 4 望 